\title{
Standard Cruciate-Retaining Total Knee Arthroplasty Implants can Reproduce Native Kinematics
}

\author{
David Leandro Dejtiar ${ }^{1,2}$, Laura Bartsoen ${ }^{3}$, Mariska Wesseling ${ }^{1}$, Roel \\ Wirix-Speetjens ${ }^{1}$, Jos Vander Sloten ${ }^{3}$ and Maria Angeles Perez ${ }^{2}$ \\ ${ }^{1}$ Materialise N.V., Leuven, Belgium \\ ${ }^{2}$ Universidad de Zaragoza, Zaragoza, Spain \\ ${ }^{3} \mathrm{KU}$ Leuven, Leuven, Belgium \\ davidleandro.dejtiar@materialise.be, laura.bartsoen@kuleuven.be, \\ mariska.wesseling@materialise.be, roel.wirix- \\ speetjens@materialise.be, jos.vandersloten@kuleuven.be, \\ angeles@unizar.es
}

\begin{abstract}
Total knee arthroplasty (TKA) is a common procedure that has become the standard of treatment for severe cases of knee osteoarthritis. Biomechanics and quality of movement similar to healthy were found to improve patient-reported outcomes.

In this study, an evaluated musculoskeletal model predicted ligament, contact and muscle forces together with secondary tibiofemoral kinematics. An artificial neural network applied to the musculoskeletal model searched for the optimal implant position in a given range that will minimize the root-mean-square-error (RMSE) between postTKA and native experimental tibiofemoral kinematics during a squat.

We found that, using a cruciate-retaining implant, native kinematics could be accurately reproduced (average RMSE $1.47 \mathrm{~mm}( \pm 0.89 \mathrm{~mm})$ for translations and $2.89^{\circ}$ $\left( \pm 2.83^{\circ}\right)$ for rotations between native and optimal TKA alignment). The required implant positions changes maximally $2.96 \mathrm{~mm}$ and $2.40^{\circ}$. This suggests that when using preoperative planning, off-the-shelf CR implants allow for reproducing native knee kinematics post-operatively.
\end{abstract}

\section{Introduction}

Total knee arthroplasty (TKA) is a common procedure that has become the standard of treatment for severe cases of knee osteoarthritis. While the number of TKA procedures is expected to further 
increase (Inacio et al., 2017), knee joint replacements are still associated with nearly $20 \%$ dissatisfaction rates (Kahlenberg et al., 2018).

The current state of the art uses mechanical alignment to position the implant, although this might not always result in an optimal outcome (Rivière et al., 2017). However, other approaches that consider soft tissues have been proposed (Lee et al., 2017). Taking into account patient-specific geometry, softtissues and kinematics has the potential to provide different alignment options to address the large dissatisfaction rates. Moreover, recent studies show that small variations in implant position from the mechanical alignment do not decrease implant survival rates (Abdel et al., 2018).

Biomechanics and quality of movement similar to healthy were found to improve post-operative patient-reported outcomes (Kirschberg et al., 2018; Biggs et al., 2019). Musculoskeletal modeling (MSM) allows to investigate the interplay between patient-specific biomechanics, implant alignment and soft tissues (Vanheule et al., 2017). Moreover, the combination of MSM and artificial neural networks (ANN) (Marra et al., 2017), allow to create a fast surrogate model to optimize patient-specific implant position. The aim of this study is to demonstrate the feasibility of restoring native knee kinematics using a cruciate-retaining (CR) implant system during a squat by means of MSM and ANN.

\section{Methods}

For two specimens, subject-specific MSM (Anybody Modeling System, Anybody Technology, Denmark) were developed and validated in previous work (Vanheule et al., 2017). The model included four segments: femur, patella, tibia and fibula. Soft tissues were included to stabilize the knee joint, being the anterior cruciate, posterior cruciate, lateral collateral, anterolateral and medial collateral ligaments, as well as the posterior capsule. For each specimen two models were created, a native joint model and TKA model incorporating a CR implant. The initial position of the CR implant was defined by mechanical alignment and the anterior cruciate ligament was removed, as this ligament is sacrificed during surgery. A force-dependent kinematics simultaneously predicted ligament, contact, and muscle forces together with secondary tibiofemoral and patellofemoral joint kinematics (Andersen et al., 2017). The models were used to simulate a squat motion from $20^{\circ}$ to $120^{\circ}$ of knee flexion similar to an Oxford Rig experiment.

Native kinematics were simulated and evaluated against experimentally recorded kinematics for the two specimens (Vanheule et al., 2017). Tibiofemoral kinematics were predicted for both the native and TKA joint. In the TKA model, multiple combinations of tibial and femoral implant positions were simulated to train an ANN on the relationship between implant alignment and knee kinematics. Implant positions were varied in the range from -3 to $+3 \mathrm{~mm}$ for anterior-posterior, medio-lateral and superiorinferior translations, $-6^{\circ}$ to $+6^{\circ}$ for internal-external rotations and $-3^{\circ}$ to $+3^{\circ}$ for varus-valgus and flexion-extension rotations. The trained ANN was used to find the optimal implant position, defined as the implant position that resulted in the smallest root-mean-square-error (RMSE) between native and post-TKA tibiofemoral kinematics within the specified implant position range.

Tibiofemoral kinematics were compared between estimations from the MSM with the implant positioned in mechanical alignment, the ANN optimized implant position and the native experiments from Vanheule et al.(Vanheule et al., 2017).

\section{Results}

The optimized MSM could reproduce native kinematics with a CR TKA (figure 1) with only small changes in implant position (table 1). We found an average RMSE of $1.47 \mathrm{~mm}( \pm 0.89 \mathrm{~mm})$ for translations and $2.89^{\circ}\left( \pm 2.83^{\circ}\right)$ for rotations between native and optimal TKA alignment, which is 
Standard Cruciate-Retaining Total Knee Arthroplasy Implants Can Reproduce Native... Dejtiar et al.

smaller compared to the RMSE for mechanical alignment (i.e. $2.93 \mathrm{~mm}( \pm 2.51 \mathrm{~mm})$ for translations and $3.38^{\circ}\left( \pm 1.42^{\circ}\right)$ for rotations). Only native internal-external rotation could not be reproduced with the optimal implant position, as RMSE remained at least $3.98^{\circ}$ for the optimal CR implant position.

\begin{tabular}{cccccccc}
\hline Specimen & Component & AP & ML & SI & $V V$ & FE & IE \\
\hline \multirow{2}{*}{1} & Tibia & 0.00 & -0.67 & -1.65 & 0.62 & 1.72 & -1.08 \\
& Femur & 0.09 & 0.56 & -2.41 & -1.51 & -0.19 & 0.62 \\
2 & Tibia & 1.83 & 0.22 & -2.96 & -2.40 & -0.02 & -1.43 \\
& Femur & 2.61 & -0.70 & -0.06 & -0.93 & -1.93 & -0.68 \\
\hline
\end{tabular}

Table 1: Implant position modifications, with respect to mechanical alignment, required to resemble native kinematics. Antero-posterior (AP), medio-lateral (ML), superior-inferior (SI), varus-valgus (VV), flexionextension (FE) and internal-external (IE).
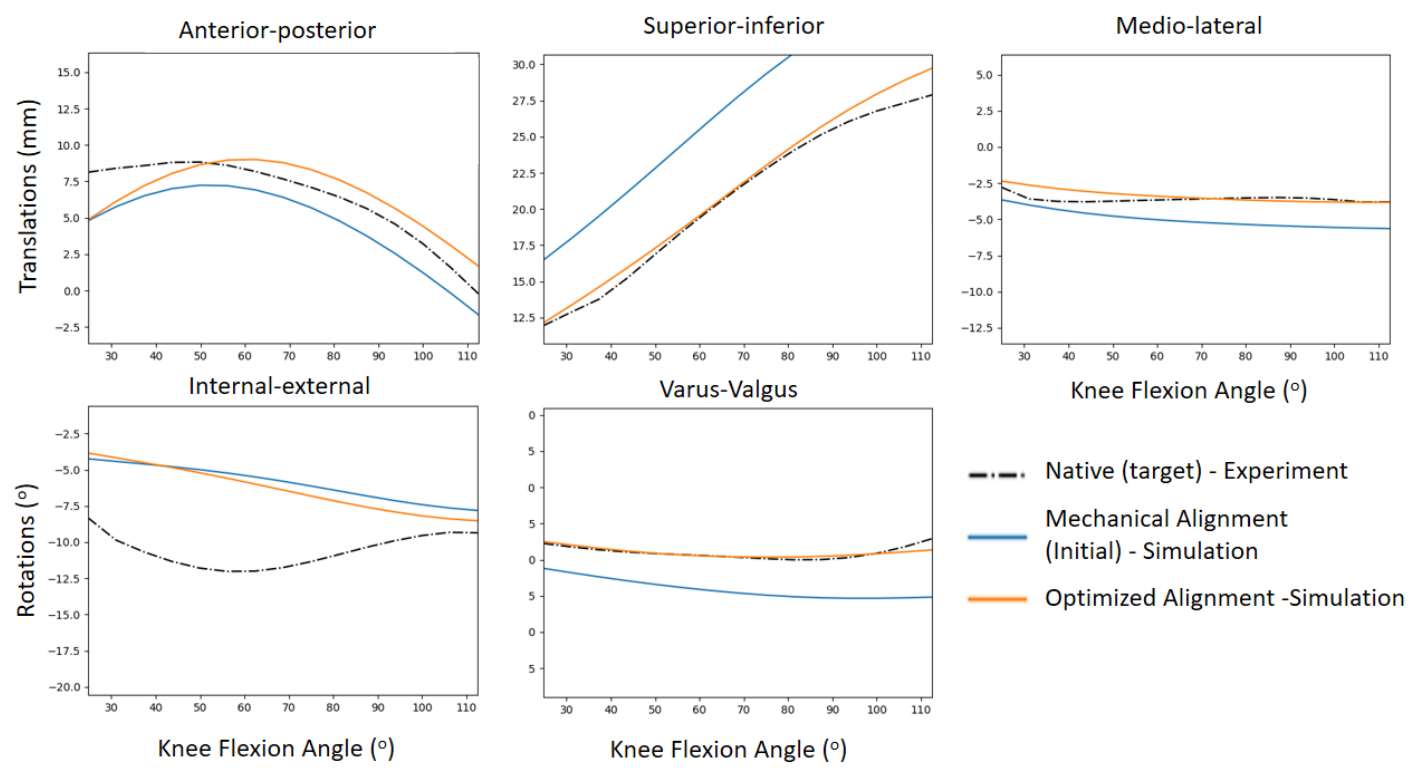

Figure 1: tibiofemoral kinematics during a squat from 20 to 120 degrees of knee flexion for specimen 1.

\section{Discussion}

In this study, we demonstrated that when using pre-operative planning, native kinematics can be reproduced with a standard CR implant, requiring only small changes in implant position. In addition, the proposed method allows for adoption to other alignment strategies, such as optimizing to native ligament elongations.

The presented work takes into account tibiofemoral and patellofemoral kinematics together with soft-tissues to position the implant, with the aim to reproduce native kinematics post-TKA. Restoring native kinematics allows for a more normal gait pattern post-TKA (Blakeney et al., 2019), which may result in improved patient-reported outcome measures (Kirschberg et al., 2018; Biggs et al., 2019). For the specimens studied, the required implant position changes were maximally $2.96 \mathrm{~mm}$ and $2.40^{\circ}$. These required changes do not represent large variations that could compromise implant survivorship (Abdel et al., 2018).

In conclusion, our method represents a powerful pre-operative planning method to restore native kinematics using a standard CR implant. The ability to automatically predict the optimal patient-specific 
Standard Cruciate-Retaining Total Knee Arthroplasy Implants Can Reproduce Native... Dejtiar et al.

implant position while taking into account the soft tissue, is an important step towards the improvement of patient-reported outcome measures. Further research will focus on extending the number of cases in our experiments as well determining the patient profile that benefits most from this planning approach.

\section{Acknowledgment}

This project has received funding from the European Union's Horizon 2020 research and innovation program under the Marie Sklodowska-Curie grant agreement No 722535.

\section{References}

Abdel, M. P. et al. (2018) 'Effect of postoperativemechanical axis alignment on survival and functional outcomes of modern total knee arthroplasties with cement: A concise follow-up at 20 years', Journal of Bone and Joint Surgery - American Volume, 100(6), pp. 472-478. doi: 10.2106/JBJS.16.01587.

Andersen, M. S. et al. (2017) 'Introduction to Force-Dependent Kinematics: Theory and Application to Mandible Modeling', Journal of Biomechanical Engineering, 139(9), p. 091001. doi: 10.1115/1.4037100.

Biggs, P. R. et al. (2019) 'Correlations between patient-perceived outcome and objectivelymeasured biomechanical change following Total Knee Replacement', Gait and Posture. Elsevier, 70(December 2018), pp. 65-70. doi: 10.1016/j.gaitpost.2019.02.028.

Blakeney, W. et al. (2019) 'Kinematic alignment in total knee arthroplasty better reproduces normal gait than mechanical alignment', Knee Surgery, Sports Traumatology, Arthroscopy. Springer Berlin Heidelberg, 27(5), pp. 1410-1417. doi: 10.1007/s00167-018-5174-1.

Inacio, M. C. S. et al. (2017) 'Projected increase in total knee arthroplasty in the United States - an alternative projection model', Osteoarthritis and Cartilage. Elsevier Ltd, 25(11), pp. 1797-1803. doi: 10.1016/j.joca.2017.07.022.

Kahlenberg, C. A. et al. (2018) 'Patient Satisfaction After Total Knee Replacement: A Systematic Review', HSS Journal, 14(2), pp. 192-201. doi: 10.1007/s11420-018-9614-8.

Kirschberg, J. et al. (2018) 'Normalized gait analysis parameters are closely related to patientreported outcome measures after total knee arthroplasty', Archives of Orthopaedic and Trauma Surgery. Springer Berlin Heidelberg, 138(5), pp. 711-717. doi: 10.1007/s00402-018-2891-3.

Lee, Y. S. et al. (2017) 'Kinematic alignment is a possible alternative to mechanical alignment in total knee arthroplasty', Knee Surgery, Sports Traumatology, Arthroscopy, 25(11), pp. 3467-3479. doi: 10.1007/s00167-017-4558-y.

Marra, M. A. et al. (2017) 'Evaluation of a Surrogate Contact Model in Force-Dependent Kinematic Simulations of Total Knee Replacement', Journal of Biomechanical Engineering, 139(8), p. 081001. doi: $10.1115 / 1.4036605$.

Rivière, C. et al. (2017) 'Alignment options for total knee arthroplasty: A systematic review', Orthopaedics and Traumatology: Surgery and Research. Elsevier Masson SAS, 103(7), pp. 10471056. doi: 10.1016/j.otsr.2017.07.010.

Vanheule, V. et al. (2017) 'Evaluation of predicted knee function for component malrotation in total knee arthroplasty', Medical Engineering and Physics. Elsevier Ltd, 40, pp. 56-64. doi: 10.1016/j.medengphy.2016.12.001. 